\title{
Escala de Estilos de Aprendizagem em Situações de Uso de Tecnologias: Estrutura Interna
}

\author{
Rodrigo Hipólito Roza ${ }^{1}$, Tatiana de Cassia Nakano, Solange Muglia Wechsler \\ Pontifícia Universidade Católica de Campinas, Campinas-SP, Brasil \\ Ricardo Primi \\ Universidade São Francisco, Campinas-SP, Brasil
}

\section{RESUMO}

Com a finalidade de buscar evidências de validade baseadas na estrutura interna da Escala de Estilos de Aprendizagem em Situações de Uso de Tecnologias, 378 estudantes universitários, provenientes de diferentes instituições de ensino superior e oito cursos, responderam ao instrumento. A aplicação da Análise Fatorial Exploratória, baseada na matriz de correlações tetracóricas dos itens, permitiu a identificação de quatro fatores: Teórico-Reflexivo, Pragmático, Ativo Individual, Ativo Interpessoal. A precisão da escala também foi estimada, por meio do alfa de Cronbach. Os valores oscilaram entre 0,70 e 0,80, tendo sido considerados adequados. Novos estudos de investigação das qualidades psicométricas da escala são recomendados.

Palavras-chave: estilo de aprendizagem; tecnologia da informação e comunicação; avaliação psicológica; análise fatorial; estrutura interna.

\section{ABSTRACT - Scale of Learning Styles in Technology Use Situations: Internal Structure}

In order to find validity evidence based on the internal structure of the Scale of Learning Styles in Technology Use Situations, 378 university students, from different institutions of higher education and eight majors, responded to the instrument. Exploratory Factor Analysis, based on the tetrachoric correlations matrix of the items, allowed the identification of four factors: Theoretical-Reflexive, Pragmatic, Individual Active, Interpersonal Active. The scale's accuracy was also estimated using the Cronbach's alpha. Values ranged from 0.70 to 0.80 and were considered adequate. New research studies on the psychometric qualities of the scale are recommended. Keywords: learning style; information and communication technology; psychological evaluation; factor analysis; internal structure.

\section{RESUMEN - Escala de Estilos de Aprendizaje en Situaciones de Uso de Technologías: Estructura Interna}

Con el fin de buscar evidencias de validez basadas en la estructura interna de la Escala de Estilos de Aprendizaje en Situaciones de Uso de Tecnologías, 378 estudiantes universitarios, procedentes de diferentes instituciones y ocho cursos, respondieron el instrumento. La aplicación del Análisis Factorial Exploratorio, basado en la matriz de correlaciones tetracóricas de los ítems, permitió la identificación de cuatro factores: Teórico-Reflexivo, Pragmático, Activo Individual, Activo Interpersonal. La precisión de la escala también fue estimada, por medio de alfa de Cronbach. Los valores oscilaron entre 0,70 y 0,80 y se consideraron adecuados. Se recomiendan nuevos estudios de investigación de las cualidades psicométricas de la escala.

Palabras clave: estilo de aprendizaje; tecnología de la información y comunicación; evaluación psicológica; análisis factorial; estructura interna.

A compreensão dos estilos de aprendizagem mostra-se como um recurso importante para auxiliar o indivíduo em seu processo de aprendizagem. Sua relevância se justifica ainda mais diante da atual realidade social, afetada pela emergência e uso das Tecnologias da Informação e Comunicação (TICs) e, consequentemente, pelas diferentes modalidades de ensino e aprendizagem resultantes.

As últimas décadas foram marcadas por profundas transformações sociais, econômicas, políticas e culturais, decorrentes, em grande parte, dos avanços das tecnologias da informação e comunicação (Castells \& Himanen, 2014; Heylighen \& Lenartowicz, 2017; Roza, 2017), que incluem, dentre outros recursos, os computadores, seus aplicativos, as redes computacionais e a Internet. Seus impactos representam novos desafios na relação com a informação e o conhecimento, mas também novas oportunidades e possibilidades de aprendizagem, maior acessibilidade e flexibilidade (Al-Azawei, Parslow, \& Lundqvist, 2017). A tecnologia aplicada ao contexto educacional oferece uma variedade de maneiras inovadoras de envolver os alunos dentro e fora da sala de 
aula, por meio, por exemplo, do incentivo ao gerenciamento do processo de ensino-aprendizado através de atividades individualizadas (Sarkar, Ford, \& Manzo, 2017). Tamanha a relevância da tecnologia na educação, que um estudo de metanálise, realizado por Chauan (2017), após consulta a 122 artigos provenientes de periódicos com revisão por pares, indicou um efeito de tamanho médio da tecnologia na efetividade da aprendizagem em estudantes do ensino fundamental.

No entanto, tais recursos tecnológicos não podem ser considerados isoladamente na educação. Nesse contexto, o indivíduo deve ser reconhecido como elemento ativo do processo de aprendizagem, que, como tal, apresenta diferenças individuais no modo de aprender, maneiras preferenciais de acessar a informação e adquirir conhecimento. Na Psicologia, o modo preferencial de um indivíduo aprender encontra aporte teórico sob o conceito de estilos de aprendizagem (Oliveira, Santos, \& Scacchetti, 2017; Roza \& Wechsler, 2017a; Santos \& Wechsler, 2008).

Definidos como um conjunto de características biológicas e de desenvolvimento que afetam a forma como os indivíduos aprendem (Dunn, Beaudry, \& Klavas, 2002), os estilos de aprendizagem são os modos característicos, relativamente estáveis, pelos quais um indivíduo adquire, percebe, conserva, interage e responde aos ambientes de aprendizagem (Alonso, Gallego, \& Honey, 1997; Felder \& Henriques, 1995). Sternberg e Grigorenko (1997) já apontavam que os estilos vinham sendo tradicionalmente vistos como sendo de potencial importância para a aprendizagem.

O conceito de estilo de aprendizagem, segundo Kolb e Kolb (2005) refere-se a diferenças individuais de aprendizagem, as quais apresentam, como base, as preferências individuais no ciclo de aprendizagem, influenciadas por variáveis hereditárias, experiências particulares de vida e demandas presentes no ambiente. O estilo de aprendizagem compreende o modo particular que cada indivíduo emprega para a aquisição de experiências e conhecimento (Santos \& Wechsler, 2008). Por tal motivo, um mesmo método de ensino pode se mostrar eficaz para algumas pessoas e ineficaz para outras.

Os estilos de aprendizagem são de fato diferentes, não existindo um melhor ou pior que o outro (Dunn et al., 2002). Historicamente, diversas teorias e diversos modelos sobre estilos de aprendizagem foram desenvolvidos ao longo das últimas décadas, podendo-se citar, como exemplos, os estudos de Dunn e Dunn (1978), Kolb (1984), Felder e Silverman (1988), Mumford e Honey (1992), Alonso, Gallego e Honey (1997), dentre outros. Uma síntese dos modelos é apresentada na Tabela 1.

Tabela 1

Modelos de Estilos de Aprendizagem

\begin{tabular}{|c|c|c|}
\hline Autor & Elementos constituintes/dimensões & Estilos de aprendizagem \\
\hline Dunn e Dunn (1978) & $\begin{array}{l}\text { Elementos ambientais, emocio- } \\
\text { nais, sociológicos, fisiológicos e } \\
\text { psicológicos. }\end{array}$ & \\
\hline Kolb (1984) & $\begin{array}{l}\text { Experiência concreta, observação } \\
\text { reflexiva, conceituação abstrata e } \\
\text { experimentação ativa. }\end{array}$ & $\begin{array}{l}\text { divergente } \\
\text { convergente } \\
\text { assimilador } \\
\text { acomodador }\end{array}$ \\
\hline Felder e Silverman (1988) & $\begin{array}{l}\text { Percepção, entrada, processamento } \\
\text { e compreensão. }\end{array}$ & $\begin{array}{l}\text { sensorial x perceptivo } \\
\text { visual x verbal } \\
\text { ativo x reflexivo } \\
\text { sequencial x global }\end{array}$ \\
\hline Mumford e Honey (1992) & & $\begin{array}{l}\text { ativo } \\
\text { reflexivo } \\
\text { teórico } \\
\text { pragmático }\end{array}$ \\
\hline Alonso et al. (1997) & & $\begin{array}{l}\text { ativo } \\
\text { reflexivo } \\
\text { teórico } \\
\text { pragmático }\end{array}$ \\
\hline
\end{tabular}

Devido ao fato da escala, foco do estudo aqui apresentado, basear-se principalmente no modelo de Alonso et al. (1997) e desse ter se originado do modelo de Mumford e Honey, eles serão melhor detalhados. Estes últimos autores desenvolveram uma versão simplificada do modelo de Kolb (1984). Os autores destacam quatro estilos: ativo, reflexivo, teórico e pragmático. Nessa concepção, pessoas com estilo de aprendizagem ativo preferem atividades novas e desafiadoras, bem como aprendem melhor por meio de experiência e trabalho com outras 
pessoas. Já aquelas com estilo reflexivo preferem ver as coisas a partir diferentes perspectivas com uma oportunidade de planejar de forma antecipada, prestar atenção em detalhes e examinar significados. Pessoas com estilo teórico conseguem aprender melhor por meio de modelos, teorias ou conceitos, bem como analisando ideias de forma lógica em ambientes estruturados. Pessoas com estilo pragmático possuem mais facilidade de aprender através de aplicações práticas e imediatas de atividades, com uma relação clara entre o assunto tratado e aplicações da vida real (Honey \& Mumford, 1999).

Alonso et al. (1997), com base nos estudos de Honey e Mumford, também consideram a existência dos estilos ativo, reflexivo, teórico e pragmático. Adicionalmente, os autores apresentam uma lista de características para determinar o campo de habilidades de cada um dos estilos de aprendizagem. Nessa lista, as características principais do estilo ativo são animador, improvisador, descobridor, arriscado e espontâneo. Do estilo reflexivo, destacam-se os adjetivos ponderado, minucioso, receptivo, analítico e exaustivo. Do estilo teórico, os descritores comumente utilizados são metódico, lógico, objetivo, crítico e estruturado. Do estilo pragmático, por sua vez, as características principais são experimentador, prático, direto, eficaz e realista.

Ainda que diferentes modelos teóricos sejam encontrados na literatura científica internacional, o que se faz notar é que a temática dos estilos de aprendizagem ainda vem sendo pouco explorada no Brasil. Silva e Wechsler (2010) analisaram a produção científica brasileira sobre estilos de aprendizagem em teses, dissertações e artigos, com as palavras-chave "estilos de aprendizagem" e "estilos de aprender", na base de dados da Coordenação de Aperfeiçoamento de Pessoal de Nível Superior (CAPES), no período entre 1990 e 2010. No total, 59 trabalhos foram encontrados. Os artigos foram buscados, no período de 1990 e 2010, em duas bases de dados da Biblioteca Virtual em Saúde e Psicologia (BVSPsi): Biblioteca Científica Eletrônica On-line (SciELO Scientific Eletronic Library Online) e Periódicos Eletrônicos em Psicologia (PePSIC). As buscas por artigos resultaram em 10 publicações. Os resultados mostraram que, nas teses e dissertações, a temática mais estudada foi estilos de aprendizagem no ensino superior, enquanto que, nos periódicos, os estilos de aprender e as estratégias de ensino foram predominantes. As autoras também apontaram que os dados analisados revelam a necessidade de novos estudos, visto que, no período de 20 anos, somente 69 trabalhos foram recuperados, indicando uma média de 3,45 trabalhos por ano.

De modo similar, Mendes e Bottentuit Junior (2015) realizaram uma revisão sistemática das teses e das dissertações sobre estilos de aprendizagem, disponíveis na base de dados da CAPES e do Instituto Brasileiro de Informação em Ciência e Tecnologia (IBICT), no período de 2010 e 2014, com a palavra-chave "estilos de aprendizagem”. As buscas resultaram em 28 trabalhos. Apesar de identificarem a existência de pesquisas com temáticas que relacionam estilos de aprendizagem a tecnologias digitais e também a educação a distância, os autores destacam que nenhuma das pesquisas analisadas trabalha com a perspectiva dos estilos de aprendizagem no uso do espaço virtual e sugerem essa perspectiva em futuros estudos.

O que se pode ver é que os estilos de aprendizagem vêm sendo definidos de formas diferentes, de acordo com a teoria que os embasa (Dunn, DeBello, Brennan, Krimsky, \& Murrain, 1981; Sternberg \& Grigorenko, 1997). Também pode ser ressaltado o fato de que tais estilos têm sido definidos com base em uma abordagem de aprendizagem tradicional, de modo a desconsiderar as novas formas de ensino e aprendizagem, notadamente a adoção de novas tecnologias nesse processo. Embora os estilos de aprendizagem sejam aplicados em diversos contextos envolvendo recursos tecnológicos, como e-learning (Deborah, Baskaran, \& Kannan, 2014; Palomino \& Rangel, 2015), ambientes virtuais de aprendizagem (Kurilovas, Kubilinskiene, \& Dagiene, 2014; Dascalu et.al., 2015) e gamificação na educação (Buckley \& Doyle, 2017), poucas pesquisas os definem especificamente em situações de uso de tecnologias. Torna-se importante citar ainda a possibilidade de uso da informação acerca dos estilos de aprendizagem para a melhoria, não só do processo de ensino-aprendizagem dos estudantes em geral, mas também de minorias, como no caso de pessoas com quadro de dislexia (Benmarrakchi, Kafi, Elhore, \& Haie, 2017), surdez ou problemas de audição (Lynn et al., 2017; Shah, \& Gathoo, 2017), deficientes visuais e cegos (Campbell, 2017).

Dentre as pesquisas que abordam os estilos de aprendizagem em situações de uso de tecnologias, pode ser citado o estudo de Barros (2009), que investigou o modo como se aprende e ensina no espaço virtual. Segundo a autora, quatro tendências podem ser notadas no uso do espaço virtual: participativo, busca e pesquisa, estruturação e planejamento, ação concreta e produção. O estilo de uso participativo no espaço virtual tem como elemento central da aprendizagem a participação, em que o indivíduo deve ter a ambiência do espaço virtual. As metodologias e materiais para indivíduos com esse estilo devem priorizar o contato com grupos on-line e solicitar buscas por situações on-line, execução de trabalhos em grupo, envolvimento em fóruns de discussão e realização de ações com os materiais desenvolvidos. $\mathrm{O}$ estilo de uso de busca e pesquisa no espaço virtual possui a necessidade de realizar pesquisa on-line e buscar informações de todos os tipos e formatos como elemento central para a aprendizagem. O indivíduo com esse estilo aprende mediante busca, seleção e organização de conteúdo. Os materiais de aprendizagem devem ser direcionados a construções e sínteses envolvendo a pesquisa de um conteúdo. 
No estilo de uso de estruturação e planejamento no espaço virtual, o elemento central para a aprendizagem é a necessidade de realizar atividades que valorizem o emprego de aplicativos para elaborar conteúdos e tarefas de planejamento. Tais atividades devem ter, como base, teorias e fundamentos sobre o que está sendo abordado. $\mathrm{O}$ estilo de uso denominado ação concreta e produção possui, como elemento central da aprendizagem, a necessidade de realizar serviços on-line e a rapidez na execução desses serviços. $\mathrm{O}$ ato de viabilizar com rapidez é destacado como um dos eixos centrais desse estilo. Complementando esse modelo e recorrendo aos estilos de uso do espaço virtual, Barros, Okada e Kenski (2012) também conceituaram estilos de coaprendizagem como os vários modos de coaprender, envolvendo a aprendizagem em rede de modo colaborativo, interativo e participativo, sendo importante também considerar os diferentes grupos de gerações e suas particularidades em relação à aprendizagem (Urick, 2016).

A diversidade de teorias e modelos sobre estilos de aprendizagem resultou em diferentes instrumentos para avaliá-los. Cué, Rincón e García (2009) identificaram 38 instrumentos destinados a conhecer as preferências de aprendizagem. Dentre esses instrumentos e em consonância com as teorias e os modelos destacados anteriormente nesta pesquisa, os mais usualmente utilizados são o Learning Style Inventory (LSI), o Learning Style Inventory and Productivity Environmental Preference Survey Learning, Learning Styles Questionnaire (LSQ), o Index of Learning Styles (ILS) e o Cuestionario HoneyAlonso de Estilos de Aprendizaje (CHAEA). Salienta-se que nenhum deles encontra-se aprovado para uso profissional, segundo a lista do Sistema de Avaliação dos Testes Psicológicos do Conselho Federal de Psicologia. Também merece atenção o fato de que todos envolvem a compreensão tradicional de estilos de aprendizagem. Somente um instrumento volta-se à avaliação dos estilos de aprendizagem em situações que envolvem o uso de tecnologias: o questionário de Estilo de Uso do Espaço Virtual (Barros, 2009).

Tal questionário tem como base as teorias de estilos de aprendizagem de Honey e Mumford e de Alonso e Gallego e as tecnologias da informação e comunicação. Ele considera quatro estilos de uso do espaço virtual participação; busca e pesquisa; estruturação e planejamento; ação concreta e produção - sendo composto por 40 itens, respondidos dicotomicamente (Cué, Rincón, \& García, 2009), de modo similar ao Questionário HoneyAlonso de estilos de aprendizagem - CHAEA (Alonso \& Gallego, 1995).

O que se verifica é que vários estudos sobre estilos de aprendizagem foram desenvolvidos nas últimas décadas, porém uma parcela menor deles foi dedicada a questões envolvendo a tecnologia, particularmente na realidade brasileira. Dessa forma, do ponto de vista científico, é necessário ampliar o entendimento sobre estilos de aprendizagem em situações de uso das tecnologias da informação e comunicação. Considerando, portanto, a necessidade de novos estudos nacionais na temática dos estilos de aprendizagem, bem como a lacuna existente em relação aos instrumentos para sua avaliação, notadamente aqueles voltados à avaliação dos estilos de aprendizagem frente às tecnologias da informação e comunicação, o processo de construção da Escala de Estilos de Aprendizagem em Situações de Uso de Tecnologias foi iniciado. Tal instrumento já passou por estudos que constataram evidências de validade baseadas no conteúdo (Roza, Wechsler, \& Nakano, 2018), bem como sucesso nos testes pilotos de verificação do funcionamento do instrumento informatizado. De modo complementar, também contou com o apoio de um estudo sobre o uso das tecnologias por estudantes de administração (Roza \& Wechsler, 2017b).

Considerando-se que, durante o processo de construção de instrumentos, uma série de estudos voltados à investigação de suas qualidades psicométricas precisam ser conduzidos, notadamente aqueles que envolvem evidências de validade e precisão, o presente estudo foi desenvolvido, tendo como objetivo a busca por evidências de validade baseadas na estrutura interna da Escala de Estilos de Aprendizagem em Situações de Uso de Tecnologias. Adicionalmente, a precisão da escala também foi investigada. A hipótese inicial pressupôs a existência de quatro fatores, correspondentes aos estilos de aprendizagem ativo, reflexivo, teórico e pragmático.

\section{Método}

\section{Participantes}

Participaram deste estudo 378 estudantes universitários de instituições de ensino superior brasileiras, públicas e privadas, sendo 211 do gênero feminino. Os estudantes possuíam idades entre 18 e 51 anos $(M=22,72$; $D P=4,88)$, sendo provenientes dos seguintes cursos: Administração $(n=322)$, Psicologia $(n=34)$, Publicidade e Propaganda $(n=9)$, Pedagogia $(n=7)$, Engenharia de Produção $(n=2)$, Relações Públicas $(n=2)$, Direito $(n=1)$ e Educação Física $(n=1)$.

A escolha da amostra foi feita por conveniência, sendo formada por estudantes universitários de cursos e turmas que possuíam disponibilidade em participar da pesquisa. No total, foram envolvidas 14 instituições de ensino superior, sendo instituições privadas em sua maioria.

\section{Instrumento}

Como instrumento, foi utilizada a Escala de Estilos de Aprendizagem em Situações de Uso de Tecnologias. Trata-se de um instrumento informatizado, construído pelo primeiro autor deste estudo. A construção da escala foi baseada principalmente no modelo de Alonso, 
Gallego e Honey (1997) e considerou quatro estilos de aprendizagem:

- Ativo - preferências por atividades novas e desafiadoras; aprendizagem por meio de experiência e trabalho coletivo.

- Reflexivo - preferências por observação a partir diferentes perspectivas com uma forma de planejar, prestar atenção em detalhes e examinar significados.

- Teórico - aprendizagem por meio de modelos, teorias ou conceitos; análise de ideias de forma lógica em ambientes estruturados.

- Pragmático - aprendizagem por meio de aplicações práticas e imediatas de atividades, com uma relação entre o assunto tratado e aplicações reais.

A escala era composta por 40 itens, sendo dez itens por estilos de aprendizagem. Para cada item da escala, o respondente deve escolher "sim" ou "não", de acordo suas preferências de aprendizagem.

\section{Procedimentos}

Primeiramente, o projeto que contempla este estudo foi analisado e aprovado pelo Comitê de Ética em Pesquisa (CEP) da Pontifícia Universidade Católica de Campinas (CAAE: 56450716.3.0000.5481). Diretores de cursos foram contatados a fim de que a autorização para aplicação fosse obtida.

Posteriormente, os alunos foram convidados a participar, receberam explicação sobre a pesquisa e, aqueles que aceitaram, apresentaram sua concordância no Termo de Consentimento Livre e Esclarecido - TCLE, mostrado na tela inicial, antes das instruções da escala. Somente aqueles que concordassem conseguiam seguir para a próxima tela, contendo as instruções. A aplicação do instrumento ocorreu em grupos, em locais e horários previamente marcados, usualmente laboratórios de informática das instituições, com duração média de sete minutos. Os itens foram respondidos em meio eletrônico.

\section{Análise de dados}

Considerando-se que a escala foi baseada em um modelo teórico que contemplava quatro estilos e que itens foram desenvolvidos para cada um desses estilos, a análise fatorial exploratória, baseada na matriz de correlações tetracóricas dos itens, foi conduzida, visando-se obter dados acerca da sua estrutura fatorial. Buscou-se, desse modo, verificar se os dados empíricos confirmavam o modelo teórico que embasou a construção de seus itens, de maneira a determinar a quantidade e a natureza das variáveis latentes (fatores) que melhor representam as variáveis observadas.

A análise fatorial exploratória foi precedida pelo Teste de Esfericidade de Bartlett e pela Medida KaiserMeyer-Olkin (KMO), a fim de que a adequação dos dados ao tipo de análise pretendida pudesse ser examinada.
Para identificação da quantidade de fatores a serem considerados na análise fatorial exploratória, a análise paralela foi conduzida. Além disso, a precisão dos fatores foi estimada por meio do alfa de Cronbach.

\section{Resultados}

Inicialmente, o Teste de Esfericidade de Bartlett apresentou valor de $\chi^{2}=2132,88, p<0,0001$, um resultado altamente significativo, rejeitando a hipótese nula, que afirmava que a matriz de correlação era igual à matriz identidade. A medida de adequação da amostra, KMO, apresentou valor de 0,66 , indicando um número satisfatório de correlações significativas entre os itens. Ambos, portanto, atestaram a adequação dos dados à realização da análise fatorial exploratória.

Em seguida, foi realizada a análise paralela, com o objetivo de determinar a quantidade de fatores a serem considerados na análise fatorial exploratória. O screeplot da análise paralela é apresentado na Figura 1, sugerindo a adoção de cinco fatores, tomando-se como critério os fatores dos dados reais (FAActual Data) que apresentaram eigenvalue maior que 1 e maior que o respectivo eigenvalue obtido na análise paralela (FA Resampled Data).

É importante salientar que a análise paralela foi escolhida por se mostrar mais conclusiva que o critério de eigenvalue >1 (critério de Kaiser-Guttman) e o método de análise do screeplot (teste de Cattell), comumente adotados para determinação da quantidade de fatores. Baseada em um procedimento estatístico de simulação, método de Monte-Carlo, a análise paralela consiste em construir aleatoriamente um conjunto de matrizes de correlações de variáveis, com a mesma dimensionalidade dos dados reais, ou seja, com as mesmas quantidades de variáveis e de sujeitos dos dados reais (no caso do presente estudo, foram 40 variáveis e 378 sujeitos). A matriz hipotética é fatorada inúmeras vezes e a média dos eigenvalues resultantes é calculada. Os valores dos eigenvalues reais são comparados com os valores aleatórios. Desse modo, os fatores a serem retidos são aqueles com eigenvalue maior que 1 e maior que o correspondente eigenvalue obtido aleatoriamente.

Posteriormente, considerando o número de fatores identificados na análise paralela, procedeu-se a análise fatorial exploratória, baseada na matriz de correlações tetracórica dos itens, por meio da rotação varimax e fixando-se em cinco o número de fatores a serem extraídos. Para definição dos fatores, foram consideradas as cargas fatoriais acima de 0,30 . Os resultados indicaram que o quinto fator agrupou apenas três itens, uma quantidade baixa frente aos 40 itens da escala. Por isso, optou-se pela realização de uma nova análise fatorial exploratória, mas com a imposição de quatro fatores, tomando-se os mesmos critérios adotados anteriormente. A Tabela 2 apresenta os fatores resultantes, os itens e suas respectivas cargas fatoriais. $\mathrm{O}$ item 14 apresentou carga fatorial baixa em todos os fatores e, por isso, foi desconsiderado. 


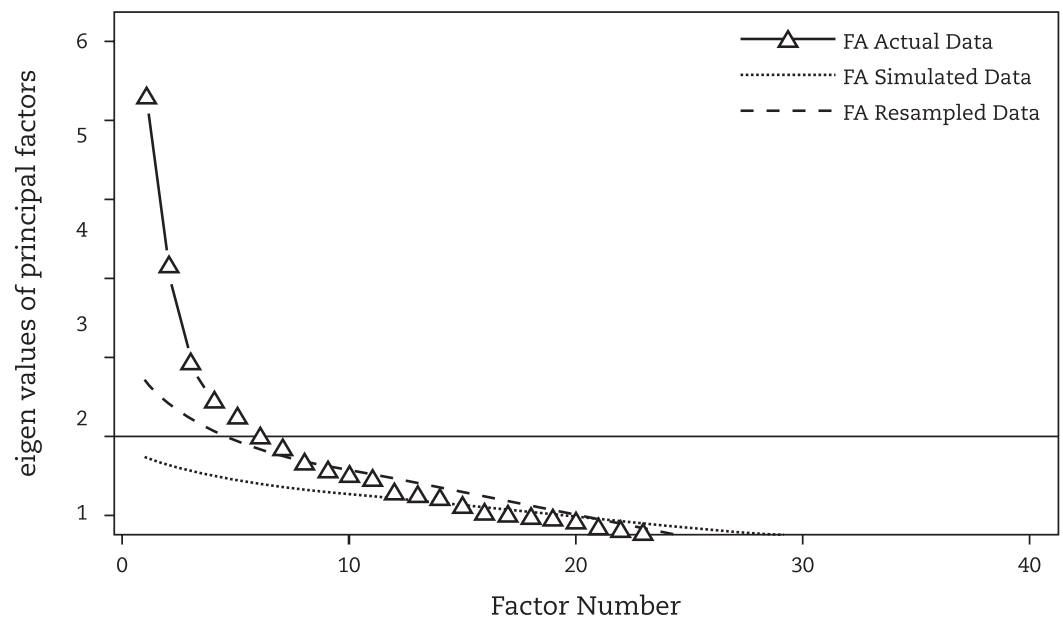

Figura 1. Scree plot da análise paralela

Tabela 2

Fatores e Cargas Fatoriais dos Itens da Escala de Estilos de Aprendizagem em Situações de Uso de Tecnologia

\begin{tabular}{|c|c|c|c|c|}
\hline Item & Fator 1 & Fator 2 & Fator 3 & Fator 4 \\
\hline 3 & 0,58 & & & \\
\hline 5 & 0,49 & & & \\
\hline 9 & 0,33 & & & \\
\hline 12 & 0,62 & & & \\
\hline 15 & 0,58 & & & \\
\hline 18 & 0,60 & & & \\
\hline 21 & 0,52 & & & \\
\hline 25 & 0,55 & & & \\
\hline 28 & 0,46 & & & \\
\hline 30 & 0,42 & & & \\
\hline 34 & 0,45 & & & \\
\hline 35 & 0,67 & & & \\
\hline 40 & 0,53 & & & \\
\hline 4 & & 0,52 & & \\
\hline 7 & & 0,75 & & \\
\hline 8 & & 0,45 & & \\
\hline 10 & & 0,57 & & \\
\hline 13 & & 0,36 & & \\
\hline 17 & & 0,69 & & \\
\hline 22 & & 0,47 & & \\
\hline 32 & & 0,50 & & \\
\hline 36 & & 0,52 & & \\
\hline 39 & & 0,65 & & \\
\hline 1 & & & 0,64 & \\
\hline 2 & & & 0,39 & \\
\hline 6 & & & 0,58 & \\
\hline 11 & & & 0,78 & \\
\hline 16 & & & 0,58 & \\
\hline 29 & & & 0,54 & \\
\hline 31 & & & 0,37 & \\
\hline 37 & & & 0,32 & \\
\hline
\end{tabular}


Tabela 2 (continuação)

Fatores e Cargas Fatoriais dos Itens da Escala de Estilos de Aprendizagem em Situações de Uso de Tecnologia

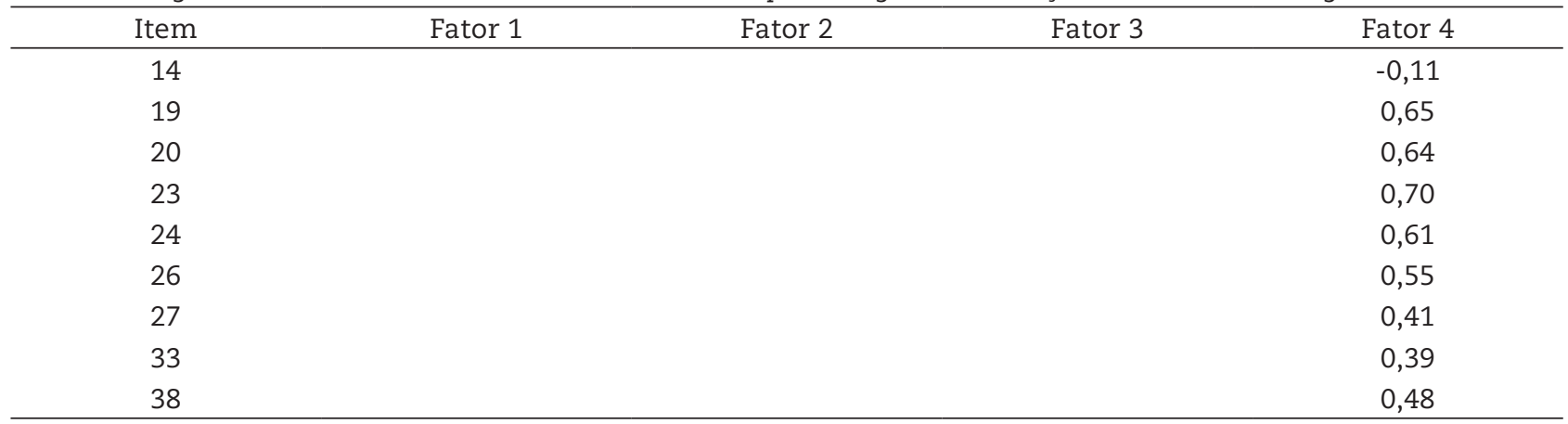

De acordo com a Tabela 2, é possível observar que o Fator 1 agrupou 13 itens com cargas fatoriais entre 0,33 e 0,67 , tendo sido nomeado de estilo teórico-reflexivo, concentrando a maior parte dos itens criados, originalmente, para representar os itens teóricos e reflexivos. Assim, indivíduos com o estilo representado nesse fator preferem aprender por meio de modelos, teorias e conceitos, bem como por meio de reflexão, usando as tecnologias da informação e comunicação no apoio a atividades analíticas e abstratas. Tal fator apresentou precisão de 0,80 (alfa de Cronbach).

O Fator 2 agrupou 10 itens com cargas entre $0,36 \mathrm{e}$ 0,75 , sendo denominado estilo pragmático, mantendo a maior parte dos itens pragmáticos originais. Indivíduos com estilo pragmático preferem aprender por meio de atividades práticas, usando as tecnologias da informação e comunicação em situações reais e na resolução de problemas concretos. $\mathrm{O}$ alfa de Cronbach desse fator foi de 0,78 .

O Fator 3 agrupou oito itens com cargas entre 0,32 e 0,78 , sendo intitulado estilo ativo individual, reuniu aspectos ativos relacionados à interação do indivíduo com as tecnologias da informação e comunicação. Indivíduos com esse estilo preferem aprender usando as tecnologias da informação e comunicação para explorar coisas novas, de forma individual. A precisão estimada, para esse fator, foi de 0,74 .

Por fim, o Fator 4 agrupou oito itens com cargas entre 0,39 e 0,70 , sendo definido como estilo ativo interpessoal, incluindo aspectos ativos relativos ao uso das tecnologias de informação e comunicação para interação do indivíduo com outros indivíduos. Esses indivíduos preferem aprender usando as tecnologias da informação e comunicação para comunicação interpessoal e realização de trabalhos colaborativos, estabelecendo relações sociais. $\mathrm{O}$ alfa de Cronbach do fator foi de 0,70.

\section{Discussão}

Este estudo concentrou-se na análise da estrutura interna da Escala de Estilos de Aprendizagem em Situações de Uso de Tecnologias, por meio da análise fatorial exploratória. Os resultados permitiram a identificação de quatro fatores, cuja análise de precisão indicou haver confiabilidade nas medidas.

Embora os itens da escala tenham sido agrupados em quatro fatores, tais fatores refletiram parcialmente o principal modelo teórico utilizado como referência para a construção dos itens (Alonso, Gallego, \& Honey, 1997). Originalmente, foram considerados quatro estilos de aprendizagem (ativo, reflexivo, teórico e pragmático), sob os quais supostamente os itens iriam se agrupar. No entanto, o Fator 1 agrupou a maioria dos itens construídos, inicialmente, para representar os estilos teórico e reflexivo. O Fator 2 agrupou a maioria dos itens construídos como estilo pragmático e, os itens construídos como ativo dividiram-se igualmente entre o Fator 3 e o Fator 4.

A Figura 2 apresenta as principais relações entre os estilos de aprendizagem, considerados originalmente (teoricamente) e os quatro fatores obtidos por meio da análise fatorial exploratória (empiricamente). Nas caixas superiores são apresentados os fatores resultantes da análise conduzida, dentro do contexto de estilos de aprendizagem em situações de uso de tecnologias e, nas caixas inferiores, nas linhas tracejadas, os estilos originais que serviram de base para o desenvolvimento dos itens, a partir do modelo proposto por Alonso, Gallego e Honey (1997).

Considerando que o Fator 1 concentrou a maioria dos itens teóricos e reflexivos originais, com predomínio dos itens teóricos, e o Fator 2 manteve a maioria dos itens pragmáticos originais, restringindo-se quase que exclusivamente a eles, observa-se que as maiores diferenças em relação ao principal modelo teórico utilizado na construção da escala estão majoritariamente nos fatores 3 e 4. O Fator 3 reuniu aspectos ativos relacionados à interação do indivíduo com as tecnologias da informação e comunicação. Já o Fator 4 agrupou aspectos ativos relativos ao uso das tecnologias da informação e comunicação para interação do indivíduo com outros indivíduos. Especificamente em relação a esses dois fatores, nota-se que seu aparecimento se justifica diante da inserção de um contexto específico em que os estilos de aprendizagem foram investigados: a situação relacionada ao uso de 
tecnologias. Assim, o que se verificou é que, três estilos tomados como base para a construção dos itens da escala se mantiveram (teórico, reflexivo e pragmático), mas agruparam-se em dois estilos, ao passo que os outros dois estilos, provenientes da análise fatorial, relacionaram-se especificamente ao contexto pretendido, representando uso de tecnologias de modo individual e para relacionamento interpessoal.

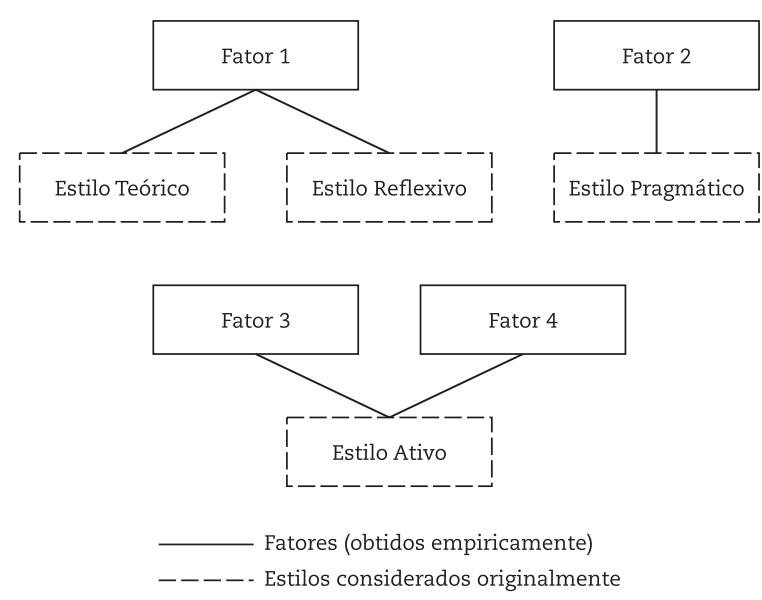

Figura 2. Principais correspondências entre os fatores e os estilos de aprendizagem considerados originalmente

Esse novo agrupamento, considerando-se a aplicação dos estilos de aprendizagem em um contexto específico, as situações de uso de tecnologias, faz sentido perante a constatação de que, usualmente, os estilos de aprendizagem foram definidos de maneiras distintas pelos pesquisadores (Dunn et. al., 1981), não havendo consenso em relação a quantos seriam e nem quais seriam, tal como apontado na Tabela 1. Do mesmo modo, a revisão dos modelos teóricos apontou para a existência de compreensão bastante diferente de seus elementos constituintes, nas diversas teorias (Sternberg \& Grigorenko, 1997). Consequentemente, esperava-se que, nesse contexto específico para a qual a escala foi construída, alguma diferença fosse encontrada em relação ao modelo teórico selecionado como base, dadas as particularidades dele.

Considerando-se ainda a grande variedade de instrumentos para avaliação de estilos de aprendizagem encontradas na literatura internacional (Cué, Rincón, \& García, 2009), a lacuna ainda existente no contexto nacional (Mendes \& Bottentuit Junior, 2015; Silva \& Wechsler, 2010) e a escassez de pesquisas de investigações dos estilos de aprendizagem em situações de uso de tecnologias, a relevância da escala em processo de desenvolvimento se justifica. Notadamente os avanços que vêm sendo incorporados nas diferentes modalidades de ensino e aprendizagem resultantes das tecnologias da informação e comunicação mostram-se uma área que merece ser melhor investigada, a fim de que a aprendizagem resultante desses novos modelos possa ser melhor planejada e direcionada, em termos de estilos preferenciais de aprendizagem (Santos \& Wechsler, 2008; Sternberg \& Grigorenko, 1997).
Em síntese, é possível destacar, portanto, que a revisão da literatura científica nacional e internacional mostra que os estilos de aprendizagem vêm sendo, historicamente, definidos com base em contextos tradicionais de aprendizagem, desconsiderando as novas formas de ensino e aprendizagem, proporcionadas pela inserção de novas tecnologias de informação e comunicação, tais como ensino a distância, por exemplo.

A proposta de um instrumento, construído para uso nesse contexto, confirmou a existência de aspectos específicos que não se encontram englobados nas teorias e instrumentos de avaliação dos estilos de aprendizagem. Dentre os quatro fatores identificados, dois deles merecem destaque, pois refletiram aspectos de interação na relação entre o indivíduo e as tecnologias da informação e comunicação: o uso individual da tecnologia e o uso da tecnologia no relacionamento interpessoal.

Dado o fato de que o estudo aqui relatado se constitui em uma das primeiras investigações das qualidades psicométricas da escala, seus resultados, ainda que positivos, somados aos anteriormente obtidos, devem ser interpretados com cautela. Principalmente se considerar que, uma das limitações do estudo, a ser citada, envolve a restrição da amostra a estudantes de nível universitário, bem como a ausência de equilíbrio entre os diferentes cursos (dada a predominância de estudantes do curso de administração).

Assim, sugere-se como sequência e desdobramento dos trabalhos de desenvolvimento da Escala de Estilos de Aprendizagem em Situações de Uso de Tecnologias, a necessidade e a importância de aprofundar a análise dos resultados empíricos obtidos, tendo em vista uma maior 
interpretação e compreensão da estrutura subjacente da escala. Do mesmo modo, a diversificação da amostra em termos de nível educacional e áreas de formação, por exemplo, é recomendada. Perante os resultados promissores encontrados até o momento, investigações de outras fontes de evidências de validade também são indicadas, a fim de que o instrumento possa, futuramente, ser disponibilizado para uso profissional.

\section{Referências}

Al-Azawei, A., Parslow, P., \& Lundqvist, K. (2017). Investigating the effect of learning styles in a blended e-learning system: An extension of the technology acceptance model (TAM). Australasian Journal of Educational Techonology, 33(2), 1-23. doi: 10.14742/ajet.2741.

Alonso, C. M., \& Gallego, D. J. (1995). Cuestionario Honey-Alonso de estilos de aprendizaje CHAEA. Recuperado de http://e-innova.inf.utfsm. cl/docs/anexo1_23_sept_2004_CHAEA1.pdf.

Alonso, C. M., Gallego, D. J., \& Honey, P. (1997). Los estilos de aprendizaje: Procedimientos de diagnostico y mejora (7a. ed.). Bilbao: Ediciones Mensajero.

Barros, D. M. V. (2009). Estilos de uso do espaço virtual: Como se aprende e se ensina no virtual? Revista Inter Ação, 34(1), 51-74. doi:10.5216/ ia.v34i1.6542

Barros, D. M. V., Okada, A., \& Kenski, V. (2012). Coletividade aberta de pesquisa: Os estilos de coaprendizagem no cenário online. Educação, Formação E Tecnologias, 5(2), 11-24. Recuperado de http://eft.educom.pt/index.php/eft/article/view/313/168

Benmarrakchi, F. E., Kafi, J. E., Elhore, A., \& Haie, S. (2017). Exploring the use of ICT in supporting dyslexic student's preferred learning styles: A preliminary evaluation. Educational and Information Techonologies, 22(6), 2939-2957. doi: 10.1007/s10639-016-9551-4

Buckley, P., \& Doyle, E. (2017). Individualizing gamification: An investigation of the impact of learning styles and personality traits on the efficacy of gamification using a prediction market. Computers \& Education, 106(2017), 43-55. doi: 10.1016/j.compedu.2016.11.009

Campbell, L. (2017). You don't need eyes to see, you need vision: Performative Pedagogy, technology and teaching arts to students with vision impairment. Journal of Pedagogic Development, 7(3), 3-12. Recuperado de https://journals.beds.ac.uk/ojs/index.php/jpd/article/ view/397/594

Castells, M., \& Himanen, P. (2014). Models of development in the global information age: constructing an analytical framework. Em M. Castells \& P. Himanen (Ed.), Reconceptualizing development in the global information age (pp. 7-25). Oxford, UK: Oxford University Press.

Chauan, S. (2017). A meta-analysis of the impact of technology on learning effectiveness of elementary students. Computers $\&$ Education, 105 , 14-30. doi: 10.1016/j.compedu.2016.11.005.

Cué, J. L. G., Rincón, J. A. S., \& García, C. M. A. (2009). Instrumentos de medición de estilos de aprendizaje. Journal of Learning Styles, 2(4), 3-21. Recuperado de https://www2.uned.es/revistaestilosdeaprendizaje/numero_4/Artigos/lsr_4_articulo_1.pdf

Deborah, L. J., Baskaran, R., \& Kannan, A. (2014). Learning styles assessment and theoretical origin in an E-learning scenario: a survey. Artificial Intelligence Review, 42(4), 801-819. doi: 10.1007/s10462-012-9344-0

Dascalu, M. I., Bodea, C. N., Moldoveanu, A., Mohora, A., Lytras, M., \& de Pablos, P. O. (2015). A recommender agent based on learning styles for better virtual collaborative learning experiences. Computers in Human Behavior, 45(2015), 243-253. doi: 10.1016/j. chb.2014.12.027

Dunn, R. S., \& Dunn, K. J. (1978). Teaching students through their individual learning styles: A practical approach. Reston: Prentice Hall.

Dunn, R., Beaudry, J. S., \& Klavas, A. (2002). Survey of research on learning styles. California Journal of Science Education, 2(2), 75-98. Recuperado de https://pdfs.semanticscholar.org/a0b1/f13e1c86bb369220b73653c28b72553f5c50.pdf.

Dunn, R., DeBello, T., Brennan, P., Krimsky, J., \& Murrain, P. (1981). Learning style researchers define differences differently. Educational Leadership, 38(5), 372-375. Recuperado de https://eric.ed.gov/?id=EJ242309.

Felder, R. M., \& Henriques, E. R. (1995). Learning and teaching styles in foreign and second language education. Foreign Language Annals, 28(1), 21-31. doi: 10.1111/j.1944-9720.1995.tb00767.x

Felder, R. M., \& Silverman, L. K. (1988). Learning and teaching styles in engineering education. Engineering Education, 78(7), 674-681. Recuperado de http://www4.ncsu.edu/unity/lockers/users/f/felder/public/Papers/LS-1988.pdf

Heylighen, F., \& Lenartowicz, M. (2017). The Global Brain as a model of the future information society: An introduction to the special issue. Technological Forecasting \& Social Change, 114(2017), 1-6. doi: 10.1016/j.techfore.2016.10.063

Honey, P., \& Mumford, A. (1999). Understanding Your Learning Styles: Learning Styles Questionnaire. Recuperado de https://www.psychpress. com.au/Psychometric/products/others/LSQ\%20Report\%20(Sample).pdf

Kolb, A. Y., \& Kolb, D. A. (2005). The Kolb learning style inventory - version 3.12005 technical specifications. Boston: Hay Resource Direct.

Kolb, D. A. (1984). Experiential learning: Experience as the source of learning and development. New Jersey: Prentice-Hall.

Kurilovas, E., Kubilinskiene, S., \& Dagiene, V. (2014). Web 3.0-Based personalization of learning objects in virtual learning environments. Computers in Human Behavior, 30(2014), 654-662. doi:10.1016/j.chb.2013.07.039

Lynn, M. A., Schley, S., Tobin, K. M., Lengyel, D., Ross, A., \& Connelly, S. J. (2017). Deaf, hard-of-hearing, and hearing students in an introductory biology course: College readiness, social learning styles and success. Journal of Developmental and Physical Disabilities, 29(1), 173-201. doi: 10.1007/s10882-016-9512-2

Mendes, A. G. L. M., \& Bottentuit Junior, J. B. (2015). Revisão sistemática da literatura (RSL) das teses e dissertações produzidas acerca dos estilos de aprendizagem. Revista EducaOnline, 9(2), 1-30. Recuperado de http://www.latec.ufrj.br/revistas/index.php?journal=educaon line\&page $=$ article\&op $=$ view\&path $\% 5 \mathrm{~B} \% 5 \mathrm{D}=706$

Mumford, A., \& Honey, P. (1992). Questions and answers on learning styles questionnaire. Industrial and Commercial Training, 24(7), 10-13. doi: 10.1108/00197859210015426

Oliveira, K. L., Santos, A. A., \& Scacchetti, F. A. (2017). Evidências de validade para uma medida de estilos de aprendizagem. Revista Psicologia: Teoria e Prática, 19(3), 159-175. doi: 10.5935/1980-6906/psicologia.v19n3p159-175 
Palomino, M., \& Rangel, J. (2015). Metodología para el desarrollo de materiales educativos audiovisuales basados en estilos de aprendizaje. Enl@ce, 12(2), 79-95. Recuperado de http://www.redalyc.org/articulo.oa?id=82340995006.

Roza, R. H. (2017). Revolução informacional e os avanços tecnológicos da informática e das telecomunicações. Ciência da Informação em Revista, 4(3), 03-11. Recuperado de http://www.seer.ufal.br/index.php/cir/article/view/3482/3029

Roza, R. H., \& Wechsler, S. M. (2017a). Análise da produção científica sobre estilos de aprendizagem e uso de tecnologias. Id on Line Revista Multidisciplinar e de Psicologia. 11(37), 13-26. doi:10.14295/idonline.v11i37.807

Roza, R. H., \& Wechsler, S. M. (2017b). O uso das tecnologias da informação e comunicação por estudantes de Administração. Competência - Revista da Educação Superior do Senac-RS, 10(2), 1-7. doi:10.24936/2177-4986.v10n2.2017.523

Roza, R. H., Wechsler, S. M., \& Nakano, T. C. (2018). Escala de estilos de aprendizagem em situações de uso de tecnologias: Busca por evidências de validade de conteúdo. Estudos Interdisciplinares em Psicologia, 9(1), 45-64. doi:10.5433/2236-6407.2018v9n1p45

Santos, E., \& Wechsler, S. (2008). Compreensão e consideração dos professores sobre estilos de aprender. Boletim - Academia Paulista de Psicologia, 28(1), 72-78. Recuperado de http://pepsic.bvsalud.org/pdf/bapp/v28n1/v28n1a09.pdf

Sarkar, N., Ford, W., \& Manzo, C. (2017). Engaging digital natives throught social learning. Systemics, Cybernetics and Informatics, $15(2), 1-4$. Recuperado de http://www.iiisci.org/journal/CV\$/sci/pdfs/EB015YQ17.pdf

Shah, J., \& Gathoo, V. (2017). Learning styles and academic achievement of children with and without hearing impairment in primary inclusive classrooms in Mumbai. Journal of Disability Management \& Special Education, 1(10), 1-9. Recuperado de http://www.jodys.info/ journal/march_2017/04_jodys_march_2017.pdf

Silva, G. D. O. L., \& Wechsler, S. M. (2010). Estilos de aprendizagem: Análise de produção científica brasileira. Journal of Learning Styles, 3(5), 146-159. Recuperado de https://www2.uned.es/revistaestilosdeaprendizaje/numero_5/articulos/lsr_5_articulo_10.pdf

Sternberg, R. J., \& Grigorenko, E. L. (1997). Are cognitive styles still in style? American Psychologist, $\overline{52} \overline{(7)}$, 700-712. doi: 10.1037/0003066X.52.7.700

Urick, M. (2016). Adapting Training to meet the preferred learning styles of diferent generations. International Journal of Training and Development, 21(1), 53-59. doi: 10.1111/ijtd.12093.

\section{Sobre os autores}

Rodrigo Hipólito Roza é graduado em Ciência da Computação, mestre em Ciência da Informação e doutor em Psicologia; pósdoutorando pela Universidade São Francisco; professor do Centro de Economia e Administração da Pontifícia Universidade Católica de Campinas.

Tatiana de Cassia Nakano é psicóloga, mestre e doutora em Psicologia, com pós-doutorado pela Universidade São Francisco; professora do curso de pós-graduação stricto sensu em Psicologia da Pontifícia Universidade Católica de Campinas.

Solange Muglia Wechsler é psicóloga, mestre e doutora em Psicologia, com pós-doutorado pela University of Georgia (EUA) e University of Buffalo (EUA); professora do curso de pós-graduação stricto sensu em Psicologia da Pontifícia Universidade Católica de Campinas.

Ricardo Primi é psicólogo, mestre e doutor em Psicologia, com pós-doutorado pela Universidade da Beira Interior (Portugal); professor do curso de pós-graduação stricto sensu em Psicologia da Universidade São Francisco. 